\title{
SUPPLEMENTS TO: FULL MATCHING APPROACH TO INSTRUMENTAL VARIABLES ESTIMATION WITH APPLICATION TO THE EFFECT OF MALARIA ON STUNTING
}

\author{
By Hyunseung Kang* Benno Kreuels ${ }^{\dagger, \ddagger}$ Jürgen MaY ${ }^{\ddagger}$ And Dylan \\ S. SMALL* \\ University of Pennsylvania*, University Medical Centre ${ }^{\dagger}$ and Bernhard \\ Nocht Institute for Tropical Medicine $e^{\ddagger}$
}

\begin{abstract}
The supplementary materials contain theoretical details of our matching method along with extended discussions about our estimator and the estimand. We also present a more detailed data analysis and additional simulation studies.
\end{abstract}

1. Supplementary Materials: Review of Notation. We adopt the notation in Section 2.1 of the main manuscript. Also, we define the effect ratio, $\lambda$, our estimator $T\left(\lambda_{0}\right)$, and $S^{2}\left(\lambda_{0}\right)$ identically as Sections 2.4 and 2.5 of the main text.

$$
\begin{aligned}
\lambda & =\frac{\sum_{i=1}^{I} \sum_{j=1}^{n_{i}} r_{1 i j}^{\left(d_{1 i j}\right)}-r_{0 i j}^{\left(d_{0 i j}\right)}}{\sum_{i=1}^{I} \sum_{j=1}^{n_{i}} d_{1 i j}-d_{0 i j}} \\
T\left(\lambda_{0}\right) & =\frac{1}{I} \sum_{i=1}^{I} V_{i}\left(\lambda_{0}\right) \\
S^{2}\left(\lambda_{0}\right) & =\frac{1}{I(I-1)} \sum_{i=1}^{I}\left\{V_{i}\left(\lambda_{0}\right)-T\left(\lambda_{0}\right)\right\}^{2}
\end{aligned}
$$

where

$$
V_{i}\left(\lambda_{0}\right)=\frac{n_{i}}{m_{i}} \sum_{j=1}^{n_{i}} Z_{i j}\left(R_{i j}-\lambda_{0} D_{i j}\right)-\frac{n_{i}}{n_{i}-m_{i}} \sum_{j=1}^{n_{i}}\left(1-Z_{i j}\right)\left(R_{i j}-\lambda_{0} D_{i j}\right)
$$

2. Supplementary Materials: Identification and Interpretation of the Effect Ratio. Let $\chi(\cdot)$ be an indicator function. Under the IV assumptions laid out in Section 2.3 of the main paper and the monotonicity assumption where $d_{1 i j} \geq d_{0 i j}$, we can identify the effect ratio and interpret

Keywords and phrases: Full matching, Instrumental variables, Malaria, Stunting, Twostage least squares 
it as the weighted average of the unit causal effect of the exposure on the treatment among individuals whose exposure was affected by the instrument. This is formalized in Proposition 1.

Proposition 1. Suppose the IV assumptions, (A1)-(A3), and SUTVA in Section 2.3 of the main manuscript holds and the exposure ranges from $0,1,2, \ldots, M$ where $M$ is an integer. Further suppose that the monotonicity assumption where $d_{1 i j} \geq d_{0 i j}$ holds for all $i, j$. Then,

$$
\begin{aligned}
\lambda & =\frac{\sum_{i=1}^{I} \sum_{j=1}^{n_{i}} E\left(R_{i j} \mid Z_{i j}=1, \mathcal{F}, \mathcal{Z}\right)-E\left(R_{i j} \mid Z_{i j}=0, \mathcal{F}, \mathcal{Z}\right)}{\sum_{i=1}^{I} \sum_{j=1}^{n_{i}} E\left(D_{i j} \mid Z_{i j}=1, \mathcal{F}, \mathcal{Z}\right)-E\left(D_{i j} \mid Z_{i j}=0, \mathcal{F}, \mathcal{Z}\right)} \\
& =\frac{\sum_{i=1}^{I} \sum_{j=1}^{n_{i}} \sum_{k=1}^{M}\left(r_{i j}^{(k)}-r_{i j}^{(k-1)}\right) \chi\left(d_{1 i j} \geq k>d_{0 i j}\right)}{\sum_{i=1}^{I} \sum_{j=1}^{n_{i}} \sum_{k=1}^{M} \chi\left(d_{1 i j} \geq k>d_{0 i j}\right)}
\end{aligned}
$$

Proof of Proposition 1. By (A3), we have

$$
\begin{aligned}
& E\left(R_{i j} \mid Z_{i j}=1, \mathcal{F}, \mathcal{Z}\right)-E\left(R_{i j} \mid Z_{i j}=0, \mathcal{F}, \mathcal{Z}\right) \\
= & r_{1 i j}^{\left(d_{1 i j}\right)}-r_{0 i j}^{\left(d_{0 i j}\right)} \\
= & \sum_{k=0}^{M} r_{1 i j}^{(k)} \chi\left(d_{1 i j}=k\right)-\sum_{k=0}^{M} r_{0 i j}^{(k)} \chi\left(d_{0 i j}=k\right) \\
= & \sum_{k=0}^{M} r_{1 i j}^{(k)}\left\{\chi\left(d_{1 i j} \geq k\right)-\chi\left(d_{1 i j} \geq k+1\right)\right\}-\sum_{k=0}^{M} r_{0 i j}^{(k)}\left\{\chi\left(d_{0 i j} \geq k\right)-\chi\left(d_{0 i j} \geq k+1\right)\right\}
\end{aligned}
$$

By $(\mathrm{A} 2), r_{1 i j}^{(k)}=r_{0 i j}^{(k)}$ for all $k$. Then, we have

$$
\begin{aligned}
& \sum_{k=0}^{M} r_{i j}^{(k)}\left\{\chi\left(d_{1 i j} \geq k\right)-\chi\left(d_{1 i j} \geq k+1\right)-\chi\left(d_{0 i j} \geq k\right)+\chi\left(d_{0 i j} \geq k+1\right)\right\} \\
= & \sum_{k=0}^{M} r_{i j}^{(k)}\left\{\chi\left(d_{1 i j} \geq k\right)-\chi\left(d_{0 i j} \geq k\right)\right\}-\sum_{k=0}^{M} r_{i j}^{(k)}\left\{\chi\left(d_{1 i j} \geq k+1\right)-\chi\left(d_{0 i j} \geq k+1\right)\right\} \\
= & \sum_{k=1}^{M} r_{i j}^{(k)}\left\{\chi\left(d_{1 i j} \geq k\right)-\chi\left(d_{0 i j} \geq k\right)\right\}-\sum_{k=1}^{M} r_{i j}^{(k-1)}\left\{\chi\left(d_{1 i j} \geq k\right)-\chi\left(d_{0 i j} \geq k\right)\right\} \\
= & \sum_{k=1}^{M}\left(r_{i j}^{(k)}-r_{i j}^{(k-1)}\right)\left\{\chi\left(d_{1 i j} \geq k\right)-\chi\left(d_{0 i j} \geq k\right)\right\}
\end{aligned}
$$

imsart-aoas ver. 2014/10/16 file: suppText-Revision3.tex date: November 10, 2015 
By monotonicity, $d_{1 i j} \geq d_{0 i j}$ for all $i, j$. Then,

$$
\begin{aligned}
& \sum_{k=1}^{M}\left(r_{i j}^{(k)}-r_{i j}^{(k-1)}\right)\left\{\chi\left(d_{1 i j} \geq k\right)-\chi\left(d_{0 i j} \geq k\right)\right\} \\
= & \sum_{k=1}^{M}\left(r_{i j}^{(k)}-r_{i j}^{(k-1)}\right) \chi\left\{\chi\left(d_{1 i j} \geq k\right)-\chi\left(d_{0 i j} \geq k\right)=1\right\} \\
= & \sum_{k=1}^{M}\left(r_{i j}^{(k)}-r_{i j}^{(k-1)}\right) \chi\left(d_{1 i j} \geq k>d_{0 i j}\right)
\end{aligned}
$$

Similarly, by (A3), the expected differences between $Z_{i j}=1$ and $Z_{i j}=0$ for the exposure $D_{i j}$ can be written as

$$
\begin{aligned}
& E\left(D_{i j} \mid Z_{i j}=1, \mathcal{F}, \mathcal{Z}\right)-E\left(D_{i j} \mid Z_{i j}=0, \mathcal{F}, \mathcal{Z}\right) \\
= & d_{1 i j}-d_{0 i j} \\
= & \sum_{k=0}^{M} k \chi\left(d_{1 i j}=k\right)-\sum_{k=0}^{M} k \chi\left(d_{0 i j}=k\right) \\
= & \sum_{k=0}^{M} k\left\{\chi\left(d_{1 i j} \geq k\right)-\chi\left(d_{1 i j} \geq k+1\right)\right\}-\sum_{k=0}^{M} k\left\{\chi\left(d_{0 i j} \geq k\right)-\chi\left(d_{0 i j} \geq k+1\right)\right\} \\
= & \sum_{k=0}^{M} k\left\{\chi\left(d_{1 i j} \geq k\right)-\chi\left(d_{1 i j} \geq k+1\right)-\chi\left(d_{0 i j} \geq k\right)+\chi\left(d_{0 i j} \geq k+1\right)\right\} \\
= & \sum_{k=0}^{M} k\left\{\chi\left(d_{1 i j} \geq k\right)-\chi\left(d_{0 i j} \geq k\right)\right\}-\sum_{k=0}^{M} k\left\{\chi\left(d_{1 i j} \geq k+1\right)-\chi\left(d_{0 i j} \geq k+1\right)\right\} \\
= & \sum_{k=1}^{M} k\left\{\chi\left(d_{1 i j} \geq k\right)-\chi\left(d_{0 i j} \geq k\right)\right\}-\sum_{k=1}^{M}(k-1)\left\{\chi\left(d_{1 i j} \geq k\right)-\chi\left(d_{0 i j} \geq k\right)\right\} \\
= & \sum_{k=1}^{M}\left\{\chi\left(d_{1 i j} \geq k\right)-\chi\left(d_{0 i j} \geq k\right)\right\}
\end{aligned}
$$

By monotonicity, we have

$$
\sum_{k=1}^{M}\left\{\chi\left(d_{1 i j} \geq k\right)-\chi\left(d_{0 i j} \geq k\right)\right\}=\sum_{k=1}^{M} \chi\left(d_{1 i j} \geq k>d_{0 i j}\right)
$$

imsart-aoas ver. 2014/10/16 file: suppText-Revision3.tex date: November 10, 2015 
Thus, we end up with

$$
\begin{aligned}
& \frac{\sum_{i=1}^{I} \sum_{j=1}^{n_{i}} E\left(R_{i j} \mid Z_{i j}=1, \mathcal{F}, \mathcal{Z}\right)-E\left(R_{i j} \mid Z_{i j}=0, \mathcal{F}, \mathcal{Z}\right)}{\sum_{i=1}^{I} \sum_{j=1}^{n_{i}} E\left(D_{i j} \mid Z_{i j}=1, \mathcal{F}, \mathcal{Z}\right)-E\left(D_{i j} \mid Z_{i j}=0, \mathcal{F}, \mathcal{Z}\right)} \\
= & \frac{\sum_{i=1}^{I} \sum_{j=1}^{n_{i}} r_{1 i j}^{\left(d_{1 i j}\right)}-r_{0 i j}^{\left(d_{0 i j}\right)}}{\sum_{i=1}^{I} \sum_{j=1}^{n_{i}} d_{1 i j}-d_{0 i j}} \\
= & \frac{\sum_{i=1}^{I} \sum_{j=1}^{n_{i}} \sum_{k=1}^{M}\left(r_{i j}^{(k)}-r_{i j}^{(k-1)}\right) \chi\left(d_{1 i j} \geq k>d_{0 i j}\right)}{\sum_{i=1}^{I} \sum_{j=1}^{n_{i}} \sum_{k=1}^{M} \chi\left(d_{1 i j} \geq k>d_{0 i j}\right)}
\end{aligned}
$$

\section{Supplementary Materials: Theoretical Properties of Test Statis-}

tic. Recall the hypothesis of interest from the main manuscript (Section $2.5)$

$$
H_{0}: \lambda=\lambda_{0}, \quad H_{a}: \lambda \neq \lambda_{0}
$$

Proposition 2 provides an asymptotic distribution for the test statistic $T\left(\lambda_{0}\right)$ in equation (2) under the null hypothesis $H_{0}$.

Proposition 2. Assume that for every $I$, (i) $n_{i}$ remains bounded and (ii) $\frac{1}{I} \sum_{i=1}^{I} \sum_{j=1}^{n_{i}} r_{1 i j}^{\left(d_{1 i j}\right)}-r_{0 i j}^{\left(d_{0 i j}\right)}$ and $\frac{1}{I} \sum_{i=1}^{I} \sum_{j=1}^{n_{i}} d_{1 i j}-d_{0 i j}$ remains fixed at $\bar{r}$ and $\bar{d} \neq 0$, respectively, so that $\bar{\lambda}=\bar{r} / \bar{d}$. In addition, we assume the following moment conditions

$$
\sum_{i=1}^{I} E\left\{V_{i}^{4}(\bar{\lambda}) \mid \mathcal{F}, \mathcal{Z}\right\}=o\left(I^{2}\right), \quad \limsup _{I \rightarrow \infty} \frac{\sum_{i=1}^{I} E\left|V_{i}(\bar{\lambda})-\mu_{i, \bar{\lambda}}\right|^{3}}{\left[\sum_{i=1}^{I} \operatorname{Var}\left\{V_{i}(\bar{\lambda})\right\}\right]^{3 / 2}}=0
$$

Then, under the null hypothesis $H_{0}: \lambda=\bar{\lambda}$, for all $t>0$,

$\limsup _{I \rightarrow \infty} P\left\{\frac{T(\bar{\lambda})}{S(\bar{\lambda})} \leq-t \mid \mathcal{F}, \mathcal{Z}\right\} \leq \Phi(-t), \quad \limsup _{I \rightarrow \infty} P\left\{\frac{T(\bar{\lambda})}{S(\bar{\lambda})} \geq t \mid \mathcal{F}, \mathcal{Z}\right\} \leq \Phi(-t)$

where $\Phi(\cdot)$ is the standard normal distribution.

To prove Proposition 2, we require the following two Lemmas. Lemma 1 characterizes the moments of the test statistics in (2). Lemma 2 derives the bias of $S^{2}\left(\lambda_{0}\right)$ in estimating the variance of $T\left(\lambda_{0}\right)$. Proof of these Lemmas are in Section 8 of the Supplementary Materials. 
Lemma 1. The expected value and the variance of the test statistic in equation (2) are

$$
\begin{aligned}
E\left\{T\left(\lambda_{0}\right) \mid \mathcal{F}, \mathcal{Z}\right\} & =\frac{1}{I}\left(\lambda-\lambda_{0}\right) \sum_{i=1}^{I} \sum_{j=1}^{n_{i}}\left(d_{1 i j}-d_{0 i j}\right) \\
\operatorname{Var}\left\{T\left(\lambda_{0}\right) \mid \mathcal{F}, \mathcal{Z}\right\} & =\frac{1}{I^{2}} \sum_{i=1}^{I} \frac{1}{n_{i}} \sum_{i=1}^{n_{i}}\left(a_{i j, \lambda_{0}}-\bar{a}_{i, \lambda_{0}}\right)^{2}
\end{aligned}
$$

where

$$
a_{i j, \lambda_{0}}=\frac{n_{i}}{m_{i}} y_{1 i j, \lambda_{0}}^{\left(d_{1 i j}\right)}+\frac{n_{i}}{n_{i}-m_{i}} y_{0 i j, \lambda_{0}}^{\left(d_{0 i j}\right)}, \quad \bar{a}_{i, \lambda_{0}}=\frac{1}{n_{i}} \sum_{i=1}^{n_{i}} a_{i j, \lambda_{0}}
$$

Lemma 2. Let $\mu_{i, \lambda_{0}}=E\left\{V_{i}\left(\lambda_{0}\right) \mid \mathcal{F}, \mathcal{Z}\right\}$ and $\mu_{\lambda_{0}}=E\left\{T\left(\lambda_{0}\right) \mid \mathcal{F}, \mathcal{Z}\right\}$. The bias of (3) in estimating the variance of the test statistic in (2) is

$$
E\left\{S^{2}\left(\lambda_{0}\right) \mid \mathcal{F}, \mathcal{Z}\right\}-\operatorname{Var}\left\{T\left(\lambda_{0}\right) \mid \mathcal{F}, \mathcal{Z}\right\}=\frac{1}{I(I-1)} \sum_{i=1}^{I}\left(\mu_{i, \lambda_{0}}-\mu_{\lambda_{0}}\right)^{2}
$$

Proof of Proposition 2. We use the same notation adopted in the proof of Lemma 2, mainly $\mu_{i, \bar{\lambda}}, \mu_{\bar{\lambda}}$, and $v_{i, \bar{\lambda}}^{2}$. In addition, let $q_{i, \bar{\lambda}}=E\left\{V_{i}^{2}(\bar{\lambda}) \mid \mathcal{F}, \mathcal{Z}\right\}$, and $v_{\bar{\lambda}}=\operatorname{Var}\{T(\bar{\lambda}) \mid \mathcal{F}, \mathcal{Z}\}$. First, $\sum_{i=1}^{I} V_{i}^{2}(\bar{\lambda}) / I$ is an unbiased estimator for $\sum_{i=1}^{I} q_{i, \bar{\lambda}} / I$. In addition,

$$
\operatorname{Var}\left\{\frac{1}{I} \sum_{i=1}^{I} V_{i}^{2}(\bar{\lambda}) \mid \mathcal{F}, \mathcal{Z}\right\} \leq \frac{1}{I^{2}} \sum_{i=1}^{I} E\left\{V_{i}^{4}(\bar{\lambda}) \mid \mathcal{F}, \mathcal{Z}\right\}
$$

By the fourth moment condition in (4), we have $\sum_{i=1}^{I} V_{i}^{2}(\bar{\lambda}) / I-\sum_{i=1}^{I} q_{i, \bar{\lambda}} / I \rightarrow$ 0 in probability. Similarly, the same fourth moment condition in (4) and the same reasoning gives $T(\bar{\lambda})-\mu_{\bar{\lambda}} \rightarrow 0$ in probability because of the growth of the variance of $T(\bar{\lambda})$ is controlled by the moment condition. Since $\mu_{\bar{\lambda}}=0$ for all $I$ under the null hypothesis, we have, by the continuous mapping theorem, $T^{2}(\bar{\lambda}) \rightarrow 0$ in probability. Combining all these convergence results, we get that for $\epsilon>0$ and $\delta>0$, there exists $I^{*}$ such that

for $I \geq I^{*}: P\left\{\frac{1}{I} \sum_{i=1}^{I} V_{i}^{2}(\bar{\lambda})-\frac{1}{I} \sum_{i=1}^{I} q_{i, \bar{\lambda}}<-\frac{\epsilon}{2}\right\}<\frac{\delta}{2}, \quad P\left\{T^{2}(\bar{\lambda})<-\frac{\epsilon}{2}\right\}<\frac{\delta}{2}$ 
and

$$
\begin{aligned}
& P\left\{I S^{2}(\bar{\lambda})-I v_{\bar{\lambda}}<-\epsilon\right\} \\
= & P\left[\frac{I}{I-1}\left\{\frac{1}{I} \sum_{i=1}^{I} V_{i}^{2}(\bar{\lambda})-T^{2}(\bar{\lambda})\right\}-I v_{\bar{\lambda}}<-\epsilon\right] \\
= & P\left[\frac{I}{I-1}\left\{\frac{1}{I} \sum_{i=1}^{I} V_{i}^{2}(\bar{\lambda})-\frac{1}{I} \sum_{i=1}^{I} q_{i, \bar{\lambda}}+\frac{1}{I} \sum_{i=1}^{I} q_{i, \bar{\lambda}}-T^{2}(\bar{\lambda})\right\}-I v_{\bar{\lambda}}<-\epsilon\right] \\
= & P\left[\frac{I}{I-1}\left\{\frac{1}{I} \sum_{i=1}^{I} V_{i}^{2}(\bar{\lambda})-\frac{1}{I} \sum_{i=1}^{I} q_{i, \bar{\lambda}}-T^{2}(\bar{\lambda})\right\}-I v_{\bar{\lambda}}+\frac{1}{I-1} \sum_{i=1}^{I} q_{i, \bar{\lambda}}<-\epsilon\right] \\
\leq & P\left[\frac{I}{I-1}\left\{\frac{1}{I} \sum_{i=1}^{I} V_{i}^{2}(\bar{\lambda})-\frac{1}{I} \sum_{i=1}^{I} q_{i, \bar{\lambda}}-T^{2}(\bar{\lambda})\right\}<-\epsilon\right] \\
\leq & \frac{\delta}{2}+\frac{\delta}{2}=\delta
\end{aligned}
$$

Stated in words, $I S^{2}(\bar{\lambda})$ will over-estimate $I v_{\bar{\lambda}}$ with high probability.

Second, under the null hypothesis $H_{0}: \lambda=\bar{\lambda}$ and from Lemma 1, $\sum_{i=1}^{I} \mu_{i, \bar{\lambda}} / I=0$. Hence, we can rewrite the test statistic as

$$
T(\bar{\lambda})=\frac{1}{I} \sum_{i=1}^{I} V_{i}(\bar{\lambda})=\frac{1}{I} \sum_{i=1}^{I}\left[V_{i}(\bar{\lambda})-\mu_{i, \bar{\lambda}}\right]
$$

where the test statistic becomes a sum of independent random variables $V_{i}(\bar{\lambda})-\mu_{i, \bar{\lambda}}$ with mean zero and variance $v_{i, \bar{\lambda}}$.

Finally, combining the two facts, under the null $H_{0}: \lambda=\bar{\lambda}$, we have

$$
\frac{T(\bar{\lambda})}{S(\bar{\lambda})}=\left[\frac{\frac{1}{I} \sum_{i=1}^{I}\left\{V_{i}(\bar{\lambda})-\mu_{i, \bar{\lambda}}\right\}}{\sqrt{\frac{1}{I^{2}} \sum_{i=1}^{I} v_{i, \bar{\lambda}}}}\right]\left\{\frac{\sqrt{\frac{1}{I^{2}} \sum_{i=1}^{I} v_{i, \bar{\lambda}}}}{\sqrt{S^{2}(\bar{\lambda})}}\right\}
$$

By conditions specified in Breiman (1992, pg 186) for the central limit theorem with non-identical distributions, the first parenthesis term converges to the standard Normal distribution. From our result about $I S_{\bar{\lambda}}^{2}$ overestimating $I v_{\bar{\lambda}}$, the second parenthesis term will be smaller than 1 with high probability. Hence, taking the sup of the entire expression, we obtain

$\limsup _{I \rightarrow \infty} P\left\{\frac{T(\bar{\lambda})}{S(\bar{\lambda})} \leq-t \mid \mathcal{F}, \mathcal{Z}\right\} \leq \Phi(-t), \quad \limsup _{I \rightarrow \infty} P\left\{\frac{T(\bar{\lambda})}{S(\bar{\lambda})} \geq t \mid \mathcal{F}, \mathcal{Z}\right\} \leq \Phi(-t)$

where $\Phi()$ is the standard normal distribution.

imsart-aoas ver. 2014/10/16 file: suppText-Revision3.tex date: November 10, 2015 
Proposition 2 provides a way to estimate the effect ratio, compute pvalues, and calculate confidence intervals. In particular, in the spirit of Hodges and Lehmann (1963), the estimator for the effect ratio, denoted as $\hat{\lambda}$, is the solution to the equation $T(\hat{\lambda}) / S(\hat{\lambda})=0$. The $95 \%$ confidence interval for the effect ratio is the solution to the equation $T(\lambda) / S(\lambda)= \pm 1.96$. Corollary 1 presents a solution to the equation $T(\hat{\lambda}) / S(\hat{\lambda})=q$ for any value of $q$.

Corollary 1. For any value $q$, the solution to $T(\lambda) / S(\lambda)=q$ is a solution to the quadratic equation $A_{2} \lambda^{2}+A_{1} \lambda+A_{0}=0$ where

$$
\begin{aligned}
& A_{2}=\bar{H}_{\cdot}^{2}-\frac{q^{2}}{I(I-1)} \sum_{i=1}^{I}\left(H_{i}-\bar{H}_{.}\right)^{2} \\
& A_{1}=-2 \bar{G} \cdot \bar{H}_{.}+\frac{2 q^{2}}{I(I-1)}\left\{\sum_{i=1}^{I}\left(G_{i}-\bar{G}_{.}\right)\left(H_{i}-\bar{H}_{.}\right)\right\} \\
& A_{0}=\bar{G}^{2}-\frac{q^{2}}{I(I-1)} \sum_{i=1}^{I}\left(G_{i}-\bar{G}_{.}\right)^{2}
\end{aligned}
$$

where

$$
\begin{aligned}
G_{i} & =\frac{n_{i}^{2}}{m_{i}\left(n_{i}-m_{i}\right)} \sum_{j=1}^{n_{i}}\left(Z_{i j}-\bar{Z}_{i .}\right)\left(R_{i j}-\bar{R}_{i .}\right) \\
H_{i} & =\frac{n_{i}^{2}}{m_{i}\left(n_{i}-m_{i}\right)} \sum_{j=1}^{n_{i}}\left(Z_{i j}-\bar{Z}_{i .}\right)\left(D_{i j}-\bar{D}_{i .}\right) \\
\bar{Z}_{i .} & =\frac{1}{n_{i}} \sum_{j=1}^{n_{i}} Z_{i j}, \quad \bar{D}_{i .}=\frac{1}{n_{i}} \sum_{j=1}^{n_{i}} D_{i j}, \quad \bar{R}_{i .}=\frac{1}{n_{i}} \sum_{j=1}^{n_{i}} R_{i j} \\
\bar{H}_{.} & =\frac{1}{I} \sum_{i=1}^{I} H_{i}, \quad \bar{G} .=\frac{1}{I} \sum_{i=1}^{I} G_{i}
\end{aligned}
$$

Proof. First, we see that $T(\lambda) / S(\lambda)=q$ implies $T^{2}(\lambda)=q^{2} S^{2}(\lambda)$. This expression can be rewritten as

$$
T^{2}(\lambda)=\frac{q^{2}}{I(I-1)} \sum_{i=1}^{I}\left(V_{i}(\lambda)-T(\lambda)\right)^{2}=\frac{q^{2}}{I(I-1)}\left\{\sum_{i=1}^{I} V_{i}^{2}(\lambda)-I T^{2}(\lambda)\right\}
$$

imsart-aoas ver. 2014/10/16 file: suppText-Revision3.tex date: November 10, 2015 
Rearranging the terms in (6), we get

$$
T^{2}(\lambda)\left(1+\frac{q^{2}}{I-1}\right)=\frac{q^{2}}{I(I-1)} \sum_{i=1}^{I} V_{i}^{2}(\lambda)
$$

Second, we can re-express $V_{i}(\lambda)$ as follows.

$$
\begin{aligned}
V_{i}(\lambda)= & \sum_{j=1}^{n_{i}}\left(\frac{n_{i}}{m_{i}}+\frac{n_{i}}{n_{i}-m_{i}}\right) Z_{i j} R_{i j}-\sum_{j=1}^{n_{i}} \frac{n_{i}}{n_{i}-m_{i}} R_{i j} \\
& -\sum_{j=1}^{n_{i}}\left(\frac{n_{i}}{m_{i}}+\frac{n_{i}}{n_{i}-m_{i}}\right) \lambda Z_{i j} D_{i j}+\sum_{j=1}^{n_{i}} \frac{n_{i}}{n_{i}-m_{i}} \lambda D_{i j} \\
= & \sum_{j=1}^{n_{i}} \frac{n_{i}^{2}}{m_{i}\left(n_{i}-m_{i}\right)} Z_{i j} R_{i j}-\left(\sum_{j=1}^{n_{i}} \frac{n_{i}}{n_{i}-m_{i}} R_{i j}\right)\left(\frac{1}{m_{i}} \sum_{j=1}^{n_{i}} Z_{i j}\right) \\
& -\sum_{j=1}^{n_{i}} \frac{n_{i}^{2}}{m_{i}\left(n_{i}-m_{i}\right)} \lambda Z_{i j} D_{i j}+\left(\sum_{j=1}^{n_{i}} \frac{n_{i}}{n_{i}-m_{i}} \lambda D_{i j}\right)\left(\frac{1}{m_{i}} \sum_{j=1}^{n_{i}} Z_{i j}\right) \\
= & \frac{n_{i}^{2}}{m_{i}\left(n_{i}-m_{i}\right)}\left(\sum_{j=1}^{n_{i}} Z_{i j} R_{i j}-\frac{1}{n_{i}} \sum_{j=1}^{n_{i}} R_{i j} \sum_{j=1}^{n_{i}} Z_{i j}\right) \\
& -\lambda \frac{n_{i}^{2}}{m_{i}\left(n_{i}-m_{i}\right)}\left(\sum_{j=1}^{n_{i}} Z_{i j} D_{i j}-\frac{1}{n_{i}} \sum_{j=1}^{n_{i}} D_{i j} \sum_{j=1}^{n_{i}} Z_{i j}\right) \\
= & \frac{n_{i}^{2}}{m_{i}\left(n_{i}-m_{i}\right)} \sum_{j=1}^{n_{i}}\left(Z_{i j}-\bar{Z}_{i .}\right)\left(R_{i j}-\bar{R}_{i .}\right)-\lambda \frac{n_{i}^{2}}{m_{i}\left(n_{i}-m_{i}\right)} \sum_{j=1}^{n_{i}}\left(Z_{i j}-\bar{Z}_{i .}\right)\left(D_{i j}-\bar{D}_{i .}\right)
\end{aligned}
$$

Immediately, we also have $V_{i}(\lambda)=G_{i}-\lambda H_{i}$. Then, we can rewrite $\sum_{i=1}^{I} V_{i}^{2}(\lambda)$ 
and $T^{2}(\lambda)$ as follows

$$
\begin{aligned}
\sum_{i=1}^{I} V_{i}^{2}(\lambda) & =\sum_{i=1}^{I}\left(G_{i}-\lambda H_{i}\right)^{2} \\
& =\sum_{i=1}^{I} G_{i}^{2}-2 \lambda \sum_{i=1}^{I} G_{i} H_{i}+\lambda^{2} \sum_{i=1}^{I} H_{i}^{2} \\
T^{2}(\lambda) & =\frac{1}{I^{2}}\left\{\sum_{i=1}^{I} V_{i}(\lambda)\right\}^{2} \\
& =\frac{1}{I^{2}}\left\{\sum_{i=1}^{I}\left(G_{i}-\lambda H_{i}\right)\right\}^{2} \\
& =\frac{1}{I^{2}}\left\{\left(\sum_{i=1}^{I} G_{i}\right)^{2}-2 \lambda \sum_{i=1}^{I} G_{i} \sum_{i=1}^{I} H_{i}+\lambda^{2}\left(\sum_{i=1}^{I} H_{i}\right)^{2}\right\}
\end{aligned}
$$

Overall, we can rewrite the equation (6) as

$$
\begin{aligned}
& \frac{1}{I^{2}}\left\{\left(\sum_{i=1}^{I} G_{i}\right)^{2}-2 \lambda \sum_{i=1}^{I} G_{i} \sum_{i=1}^{I} H_{i}+\lambda^{2}\left(\sum_{i=1}^{I} H_{i}\right)^{2}\right\}\left(1+\frac{q^{2}}{I-1}\right) \\
= & \frac{q^{2}}{I(I-1)}\left(\sum_{i=1}^{I} G_{i}^{2}-2 \lambda \sum_{i=1}^{I} G_{i} H_{i}+\lambda^{2} \sum_{i=1}^{I} H_{i}^{2}\right)
\end{aligned}
$$

Finally, we pull out the coefficients associated with $\lambda^{2}$ and $\lambda$, denoted as $A_{2}$ and $A_{1}$, respectively. The remaining term are constants and we denote them 
as $A_{0}$. All $A_{2}, A_{1}$, and $A_{0}$ are explicitly written below.

$$
\begin{aligned}
A_{2} & =\frac{1}{I^{2}}\left(\sum_{i=1}^{I} H_{i}\right)^{2}+\frac{q^{2}}{I(I-1)}\left\{\frac{1}{I}\left(\sum_{i=1}^{I} H_{i}\right)^{2}-\sum_{i=1}^{I} H_{i}^{2}\right\} \\
& =\bar{H}_{\cdot}^{2}-\frac{q^{2}}{I(I-1)} \sum_{i=1}^{I}\left(H_{i}-\bar{H}_{.}\right)^{2} \\
A_{1} & =-2\left[\frac{1}{I^{2}} \sum_{i=1}^{I} G_{i} \sum_{i=1}^{I} H_{i}+\frac{q^{2}}{I(I-1)}\left\{\frac{1}{I} \sum_{i=1}^{I} G_{i} \sum_{i=1}^{I} H_{i}-\sum_{i=1}^{I} G_{i} H_{i}\right\}\right] \\
& =-2\left[\bar{G}_{.} \cdot \bar{H}_{\cdot}-\frac{q^{2}}{I(I-1)}\left\{\sum_{i=1}^{I}\left(G_{i}-\bar{G}_{.}\right)\left(H_{i}-\bar{H}_{.}\right)\right\}\right] \\
A_{0} & =\frac{1}{I^{2}}\left(\sum_{i=1}^{I} G_{i}\right)^{2}+\frac{q^{2}}{I(I-1)}\left\{\frac{1}{I}\left(\sum_{i=1}^{I} G_{i}\right)^{2}-\sum_{i=1}^{I} G_{i}^{2}\right\} \\
& =\bar{G}_{.}^{2}-\frac{q^{2}}{I(I-1)} \sum_{i=1}^{I}\left(G_{i}-\bar{G}_{.}\right)^{2}
\end{aligned}
$$

If $q=0$ in Corollary 1, there is only one solution to the quadratic equation since

$$
A_{2} \lambda^{2}+A_{1} \lambda+A_{0}=\bar{H}_{\cdot}^{2} \lambda^{2}-2 \bar{H} \cdot \bar{G} \cdot \lambda+\bar{G}^{2}=(\bar{H} \cdot \lambda-\bar{G} \cdot)^{2}=0
$$

This gives us an explicit formula for the estimator of the effect ratio, denoted as $\hat{\lambda}$.

$$
\hat{\lambda}=\frac{\bar{G}}{\bar{H} .}=\frac{\sum_{i=1}^{I} \frac{n_{i}^{2}}{m_{i}\left(n_{i}-m_{i}\right)} \sum_{j=1}^{n_{i}}\left(R_{i j}-\bar{R}_{i .}\right)\left(Z_{i j}-\bar{Z}_{i .}\right)}{\sum_{i=1}^{I} \frac{n_{i}^{2}}{m_{i}\left(n_{i}-m_{i}\right)} \sum_{j=1}^{n_{i}}\left(D_{i j}-\bar{D}_{i .}\right)\left(Z_{i j}-\bar{Z}_{i .}\right)}
$$

4. Supplementary Materials: Sensitivity Analysis. To model this deviation from randomized assignment due to unmeasured confounders, let $\pi_{i j}=P\left(Z_{i j}=1 \mid \mathcal{F}\right)$ and $\pi_{i k}=P\left(Z_{i k}=1 \mid \mathcal{F}\right)$ for each unit $j$ and $k$ in the $i$ th matched set. The odds that unit $j$ will receive $Z_{i j}=1$ instead of $Z_{i j}=0$ is $\pi_{i j} /\left(1-\pi_{i j}\right)$. Similarly, the odds for unit $k$ is $\pi_{i k} /\left(1-\pi_{i k}\right)$. Suppose the ratio of these odds is bounded by $\Gamma \geq 1$

$$
\frac{1}{\Gamma} \leq \frac{\pi_{i j}\left(1-\pi_{i k}\right)}{\pi_{i k}\left(1-\pi_{i j}\right)} \leq \Gamma
$$

imsart-aoas ver. 2014/10/16 file: suppText-Revision3.tex date: November 10, 2015 
If unmeasured confounders play no role in the assignment of $Z_{i j}, \Gamma=1$ and $\pi_{i j}=\pi_{i k}$. That is, child $j$ and $k$ have the same probability of receiving $Z_{i j}=1$ in matched set $i$. If there are unmeasured confounders that affect the distribution of $Z_{i j}$, then $\pi_{i j} \neq \pi_{i k}$ and $\Gamma>1$. By Rosenbaum (2002), equation (8) is equivalent to

$$
P(\mathbf{Z}=\mathbf{z} \mid \mathcal{F}, \mathcal{Z})=\frac{\exp \left(\gamma \mathbf{z}^{T} \mathbf{u}\right)}{\sum_{b \in \Omega} \exp \left(\gamma \mathbf{b}^{T} \mathbf{u}\right)}
$$

where $\mathbf{u}=\left(u_{11}, u_{12}, \ldots, u_{I n_{I}}\right)$. Unfortunately, the exact probability of (9) is unknown as it depends on the vector of unobserved confounders, $\left(u_{11}, \ldots, u_{I n_{I}}\right)$. However, for a fixed $\Gamma>1$, we can obtain lower and upper bounds on (9). Furthermore, since the inference on the effect ratio $\lambda$ is derived from the distribution of $P(\mathbf{Z}=\mathbf{z} \mid \mathcal{F}, \mathcal{Z})$, these bounds can be used to compute a range of possible p-values under the null hypothesis. The range of p-values indicates the effect of unmeasured confounders on the conclusions reached by the inference on $\lambda$. If the range contains $\alpha$, the significance value, then we cannot reject the null hypothesis at the $\alpha$ level when there is an unmeasured confounder with an effect quantified by $\Gamma$.

Specifically, consider Fisher's sharp null hypothesis, $H_{0}: r_{1 i j}^{\left(d_{1 i j}\right)}=r_{0 i j}^{\left(d_{0 i j}\right)}$ for all $i=1, \ldots, n$ and $j=1, \ldots, n_{i}$. Note that this hypothesis implies the hypothesis $H_{0}: \lambda=0$. Furthermore, the test statistic in (2) simplifies to

$$
\begin{aligned}
T(0) & =\frac{1}{I} \sum_{i=1}^{I}\left\{\frac{n_{i}}{m_{i}} \sum_{j=1}^{n_{i}} Z_{i j} R_{i j}-\frac{n_{i}}{n_{i}-m_{i}} \sum_{j=1}^{n_{i}}\left(1-Z_{i j}\right) R_{i j}\right\} \\
& =\frac{1}{I} \sum_{i=1}^{I} \frac{n_{i}^{2}}{m_{i}\left(n_{i}-m_{i}\right)} \sum_{j=1}^{n_{i}} Z_{i j} R_{i j}-\frac{1}{I} \sum_{i=1}^{I} \frac{n_{i}}{n_{i}-m_{i}} \sum_{j=1}^{n_{i}} R_{i j}
\end{aligned}
$$

Regardless of the distribution of $P(\mathbf{Z}=\mathbf{z} \mid \mathcal{F}, \mathcal{Z}), \frac{1}{I} \sum_{i=1}^{I} n_{i} /\left(n_{i}-m_{i}\right) \sum_{j=1}^{n_{i}} R_{i j}$ is a constant since $r_{1 i j}^{\left(d_{1 i j}\right)}=r_{0 i j}^{\left(d_{0 i j}\right)}$ under Fisher's sharp null hypothesis. Hence, we can use the simpler statistic, $\tilde{T}(0)$,

$$
\tilde{T}(0)=\frac{1}{I} \sum_{i=1}^{I} \frac{n_{i}}{m_{i}\left(n_{i}-m_{i}\right)} \sum_{j=1}^{n_{i}} Z_{i j} R_{i j}
$$

to test the Fisher's sharp null hypothesis. If the responses are binary, equation (10) is the sign-score test statistic for which exact bounds on p-values exist (Rosenbaum, 2002). If the responses are continuous, Gastwirth et al. (2000) and Small et al. (2009) provide an approximate bound on p-values.

imsart-aoas ver. 2014/10/16 file: suppText-Revision3.tex date: November 10, 2015 
5. Supplementary Materials: Amplification of Sensitivity Analysis. Following Rosenbaum and Silber (2009), we can also reinterpret the sensitivity parameter $\Gamma$ by considering a binary unmeasured confounder with two values $\Delta$ and $\Lambda$ where $\Delta$ and $\Lambda$ have the following property

$$
\Gamma=\frac{\Delta \Lambda+1}{\Delta+\Lambda}, \quad \Delta>0, \Lambda>0
$$

The parameter $\Lambda$ refers to the odds of having one instrument value over another. The parameter $\Delta$ refers to the odds of having one outcome over another. For each $\Gamma$, we can use equation (11) and translate the interpretation of $\Gamma$ as the combined effect an unmeasured confounder must have on the instrument, $\Lambda$, and on the outcome, $\Delta$, to change the inference.

Figure 1 shows the result of applying the amplification of $\Gamma$ by looking at the effect by unmeasured confounders on the odds of stunting and odds of inheriting HbAS over HbAA and on the inference. Specifically, the different values of $\Gamma$ in the sensitivity analysis provides us with range of possible p-values. By equation (11), each $\Gamma$ is associated with two other sensitivity parameters $\Delta$, odds of stunting, and $\Lambda$, odds of inheriting HbAS over HbAA, and can be presented as a two-dimensional plot with each axis representing $\Delta$ and $\Lambda$. For example, the point $(\Delta=1.5, \Lambda=1.5)$ on Figure 1 represents an unmeasured confounder that increases the odds of stunting and inheriting $\mathrm{HbAS}$ over $\mathrm{HbAA}$ by a factor of 1.5 and produces a p-value in between 0.025 and 0.05 , which does not contain the significance level of 0.05 . Hence, the null hypothesis would still be rejected despite having such an unmeasured confounder. In contrast, if the unmeasured confounder had an effect of $(2.0,2.0)$ specified on the plot, the null hypothesis would be retained since the p-value contains the significance level of 0.05 .

\section{Supplementary Materials: Efficiency.}

6.1. Formula for efficiency. One of the advantages of full matching is its flexibility to accommodate various sizes of matched sets. All things being equal in terms of covariate balance, we would like an estimator of the effect ratio $\lambda$ that is as efficient as possible. This is particularly the case with full matching where an unconstrained full matching can create large matched sets which reduces efficiency (Hansen, 2004). However, we can constrain full matching to increase efficiency by restricting matched sets to have a maximum number of controls and/or treated units per matched set (Hansen, 2004). This section studies the statistical efficiency of the estimator for $\lambda$ in equation (7) under different constraints on full matching.

imsart-aoas ver. 2014/10/16 file: suppText-Revision3.tex date: November 10, 2015 


\section{Sensitivity Analysis to Unmeasured Confounders}

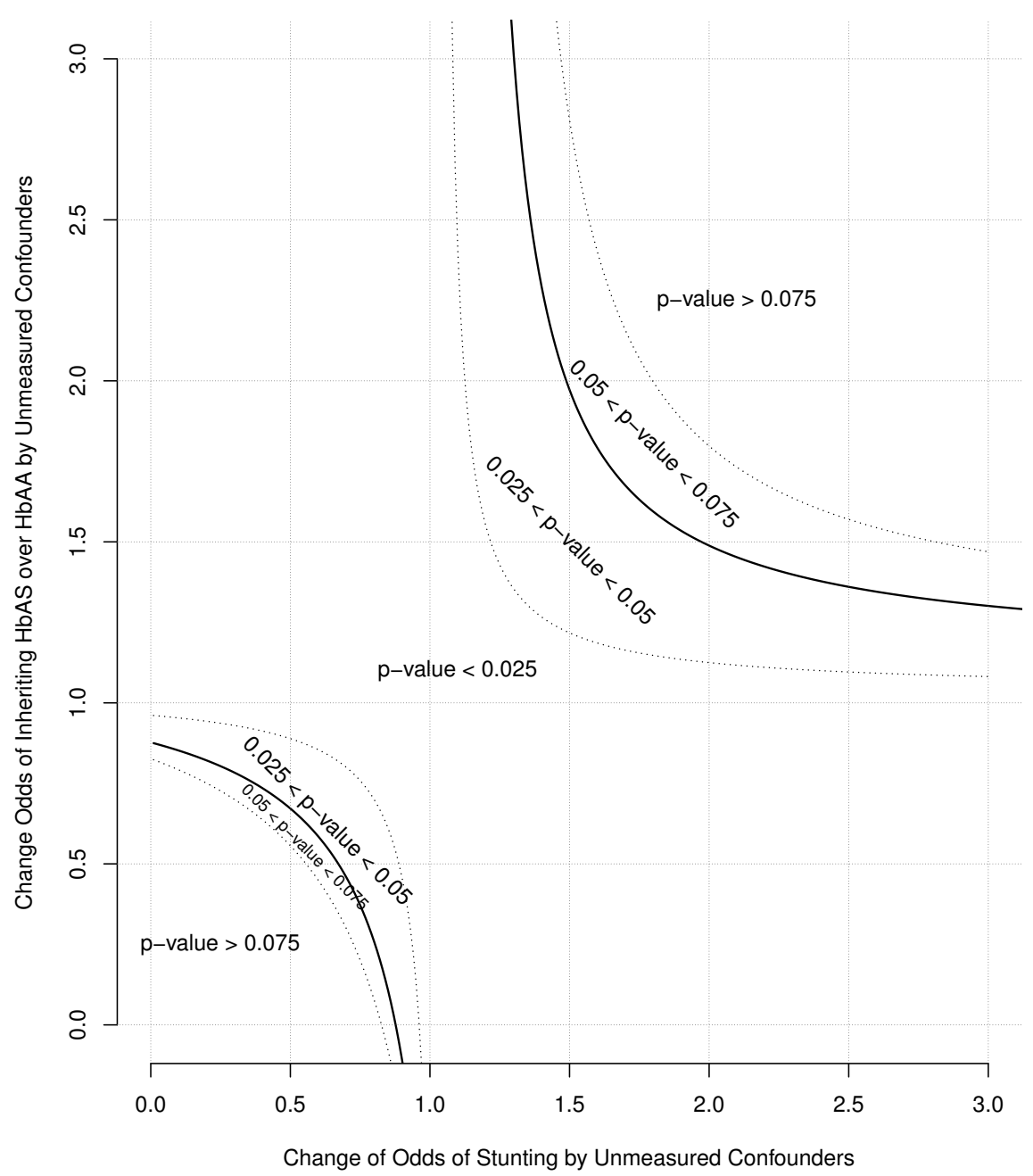

FIG 1. Amplification of sensitivity analysis. Each point on the graph represents an effect by an unmeasured confounder on the instrument (HbAS) and on the outcome (stunting) to change the inference, specifically the p-value. Points within the two bold curves correspond to effects by unmeasured confounders that will give us $p$-values $<0.05$ and points outside the two bold curves correspond to effects that will give us p-values $>0.05$, thereby retaining our null hypothesis.

imsart-aoas ver. 2014/10/16 file: suppText-Revision3.tex date: November 10, 2015 
To study the efficiency of the effect ratio estimator for different $n_{i}$ and $m_{i}$, we study a simple version of the structural equations model introduced popular in econometrics and has been used to study the properties of 2SLS, the most popular IV estimator (Wooldridge, 2010). Let $\left(R_{i j}, D_{i j}, Z_{i j}\right)$ be i.i.d. observations from an infinite population under the following model.

$$
\begin{aligned}
& R_{i j}=\alpha_{i}+\beta D_{i j}+\epsilon_{i j}, \quad E\left(\epsilon_{i j} \mid Z_{i j}\right)=0 \\
& D_{i j}=\tau_{i}+\gamma Z_{i j}+\xi_{i j}, \quad E\left(\xi_{i j} \mid Z_{i j}\right)=0
\end{aligned}
$$

with the following moment conditions.

$$
\operatorname{Var}\left(\epsilon_{i j} \mid Z_{i j}\right)=\sigma_{i, R}^{2}, \quad \operatorname{Var}\left(\xi_{i j} \mid Z_{i j}\right)=\sigma_{i, D}^{2}, \quad E\left(\epsilon_{i j} \xi_{i j} \mid Z_{i j}\right)=\sigma_{i, R D}
$$

The parameters $\alpha_{i}, i=1, \ldots, I$ measure the effect on the outcome from being in matched set $i$. The parameter $\beta$ is the effect of interest, the effect of the exposure on the outcome. Note that the treatment effect in (12) is assumed to be homogeneous for everyone, which is not assumed in the main manuscript. The parameters $\tau_{i}, i=1, \ldots, I$ measure the effect on the exposure from being in matched set $i$. The parameter $\gamma$ is the effect of the instrument on the exposure. By including $\alpha_{i}$ and $\tau_{i}$, the models (12) and (13) incorporate the matching aspect of IV estimation since each matched set $i$ have effects on $R_{i j}$ and $D_{i j}$ that are unique to that matched set.

The effect ratio, $\lambda$, is related to parameters found in standard structural equation models in (12) and (13). To illustrate this, note that the potential outcomes notation can be rewritten under the models (12) and (13) as follows.

$$
\begin{aligned}
& R_{i j}= \begin{cases}r_{1 i j}^{\left(d_{1 i j}\right)}=\alpha_{i}+\beta \tau_{i}+\beta \gamma+\beta \xi_{i j}+\epsilon_{i j} & \text { if } Z_{i j}=1 \\
r_{0 i j}^{\left(d_{0 i j}\right)}=\alpha_{i}+\beta \tau_{i}+\beta \xi_{i j}+\epsilon_{i j} & \text { if } Z_{i j}=0\end{cases} \\
& D_{i j}= \begin{cases}d_{1 i j}=\tau_{i}+\gamma+\xi_{i j} & \text { if } Z_{i j}=1 \\
d_{0 i j}=\tau_{i}+\xi_{i j} & \text { if } Z_{i j}=0\end{cases}
\end{aligned}
$$

Then, the effect ratio in (1) turns out to be

$$
\lambda=\frac{\sum_{i=1}^{I} \sum_{j=1}^{n_{i}} r_{1 i j}^{\left(d_{1 i j}\right)}-r_{0 i j}^{\left(d_{0 i j}\right)}}{\sum_{i=1}^{I} \sum_{j=1}^{n_{i}} d_{1 i j}-d_{0 i j}}=\frac{\sum_{i=1}^{I} \sum_{j=1}^{n_{i}} \beta \gamma}{\sum_{i=1}^{I} \sum_{j=1}^{n_{i}} \gamma}=\frac{\beta \gamma}{\gamma}=\beta
$$

Hence, $\lambda=\beta$ and because of this equivalence, inferences for the effect ratio provides inference for $\beta$.

Thus, the parameter $\beta$ can be estimated by the effect ratio estimator discussed in Section 3 of the Supplementary Materials, specifically equation

imsart-aoas ver. 2014/10/16 file: suppText-Revision3.tex date: November 10, 2015 
(7),

$$
\hat{\beta}=\frac{\sum_{i=1}^{I} \frac{n_{i}^{2}}{m_{i}\left(n_{i}-m_{i}\right)} \sum_{j=1}^{n_{i}}\left(Z_{i j}-\bar{Z}_{i .}\right)\left(R_{i j}-\bar{R}_{i .}\right)}{\sum_{i=1}^{I} \frac{n_{i}^{2}}{m_{i}\left(n_{i}-m_{i}\right)} \sum_{j=1}^{n_{i}}\left(Z_{i j}-\bar{Z}_{i .}\right)\left(D_{i j}-\bar{D}_{i .}\right)}
$$

Proposition 3 computes the asymptotic variance of $\hat{\beta}$ to study the efficiency of the effect ratio estimator.

Proposition 3. Suppose we have models (12) and (13) with $\gamma \neq 0$ and the third moment of $\epsilon_{i j}$ is bounded for all $i, j$. Define the following variables

$$
\begin{aligned}
J_{i} & =\sum_{j=1}^{n_{i}}\left(Z_{i j}-\bar{Z}_{i .}\right)\left(\epsilon_{i j}-\bar{\epsilon}_{i .}\right), \quad H_{i}=\sum_{j=1}^{n_{i}}\left(Z_{i j}-\bar{Z}_{i .}\right)\left(D_{i j}-\bar{D}_{i .}\right), \quad \bar{\epsilon}_{i .}=\frac{1}{n_{i}} \sum_{j=1}^{n_{i}} \epsilon_{i j} \\
s_{I}^{2} & =\sum_{i=1}^{I} \frac{n_{i}^{3}}{m_{i}\left(n_{i}-m_{i}\right)} \sigma_{i, R}^{2}
\end{aligned}
$$

Assume that (i) $Z_{i j}$ are fixed, (ii) $n_{i}$ remain bounded for all $i$, and the following moment conditions are met for $J_{i}$ and $H_{i}$

$\limsup _{I \rightarrow \infty} \frac{1}{s_{I}^{3}} \sum_{i=1}^{I} \frac{n_{i}^{6}}{m_{i}^{3}\left(n_{i}-m_{i}\right)^{3}} E\left(\left|J_{i}\right|^{3}\right)=0, \quad \sum_{i=1}^{I} \operatorname{Var}\left(\frac{n_{i}^{2}}{m_{i}\left(n_{i}-m_{i}\right)} H_{i}^{2}\right)=o\left(I^{2}\right)$

Then, the asymptotic variance of the effect ratio estimator in (7) is

$$
\sqrt{I}(\hat{\beta}-\beta) \rightarrow N\left\{0, \frac{\left(\lim _{I \rightarrow \infty} \frac{s_{I}}{\sqrt{I}}\right)^{2}}{\gamma^{2}\left(\lim _{I \rightarrow \infty} \frac{1}{I} \sum_{i=1}^{I} n_{i}\right)^{2}}\right\}
$$

Proof of Proposition 3. First, for all $i=1, \ldots, I$ and $j=1, \ldots, n_{i}$, we have

$$
Z_{i j}-\bar{Z}_{i .}= \begin{cases}1-\frac{m_{i}}{n_{i}} & \text { if } Z_{i j}=1 \\ -\frac{m_{i}}{n_{i}} & \text { if } Z_{i j}=0\end{cases}
$$

Furthermore,

$$
\sum_{j=1}^{n_{i}}\left(Z_{i j}-\bar{Z}_{i .}\right)=0, \quad \sum_{j=1}^{n_{i}}\left(Z_{i j}-\bar{Z}_{i .}\right)^{2}=\frac{m_{i}\left(n_{i}-m_{i}\right)}{n_{i}}
$$


Second, for fixed $Z_{i j}$, we have the following expected values for $J_{i}$

$$
\begin{aligned}
E\left(J_{i}\right) & =0 \\
E\left(J_{i}^{2}\right) & =\operatorname{Var}\left\{\sum_{j=1}^{n_{i}}\left(Z_{i j}-\bar{Z}_{i .}\right)\left(\epsilon_{i j}-\bar{\epsilon}_{i .}\right)\right\} \\
& =\sum_{j=1}^{n_{i}}\left(Z_{i j}-\bar{Z}_{i .}\right)^{2} \operatorname{Var}\left(\epsilon_{i j}-\bar{\epsilon}_{i .}\right)+\sum_{j, k}\left(Z_{i j}-\bar{Z}_{i .}\right)\left(Z_{i k}-\bar{Z}_{k .}\right) \operatorname{Cov}\left(\epsilon_{i j}-\bar{\epsilon}_{i .}, \epsilon_{i k}-\bar{\epsilon}_{i .}\right) \\
& =\left(1-\frac{1}{n_{i}}\right) \sigma_{i, R}^{2} \sum_{j=1}^{n_{i}}\left(Z_{i j}-\bar{Z}_{i .}\right)^{2}-\frac{1}{n_{i}} \sigma_{i, R}^{2} \sum_{j, k}\left(Z_{i j}-\bar{Z}_{i .}\right)\left(Z_{i k}-\bar{Z}_{k .}\right) \\
& =\sigma_{i, R}^{2} \sum_{j=1}^{n_{i}}\left(Z_{i j}-\bar{Z}_{i .}\right)^{2}-\frac{1}{n_{i}} \sigma_{i, R}^{2}\left\{\sum_{j=1}^{n_{i}}\left(Z_{i j}-\bar{Z}_{i .}\right)\right\}^{2} \\
& =\sigma_{i, R}^{2} \frac{m_{i}\left(n_{i}-m_{i}\right)}{n_{i}}
\end{aligned}
$$

For the third moment, for each $i$, let $k_{1}, \ldots, k_{n_{i}}$ be non-negative integers and define the multinomial coefficient as follows.

$$
\left(\begin{array}{c}
3 \\
k_{1}, \ldots, k_{n_{i}}
\end{array}\right)=\frac{3 !}{k_{1} ! \cdots k_{n_{i}} !}
$$

Then, we have

$$
\begin{aligned}
E\left(\left|J_{i}^{3}\right|\right) & =E\left|\left\{\sum_{j=1}^{n_{i}}\left(Z_{i j}-\bar{Z}_{i .}\right)\left(\epsilon_{i j}-\bar{\epsilon}_{i .}\right)\right\}^{3}\right| \\
& =E\left|\sum_{k_{1}+\cdots+k_{n_{i}}=3}\left(\begin{array}{c}
3 \\
k_{1}, \ldots, k_{n_{i}}
\end{array}\right) \prod_{j=1}^{n_{i}}\left\{\left(Z_{i j}-\bar{Z}_{i .}\right)\left(\epsilon_{i j}-\bar{\epsilon}_{i .}\right)\right\}^{k_{j}}\right| \\
& \leq \sum_{k_{1}+\cdots+k_{n_{i}}=3}\left(\begin{array}{c}
3 \\
k_{1}, \ldots, k_{n_{i}}
\end{array}\right) \prod_{j=1}^{n_{i}}\left|Z_{i j}-\bar{Z}_{i .}\right|^{k_{j}} E\left|\epsilon_{i j}-\bar{\epsilon}_{i .}\right|^{k_{j}}<\infty
\end{aligned}
$$

because third moments exist and are bounded for all $\epsilon_{i j}$ and $n_{i}$ is bounded.

Third, based on these moment calculations, it immediately follows that

$E\left[\sum_{i=1}^{I}\left\{\frac{n_{i}^{2}}{\left(m_{i}\right)\left(n_{i}-m_{i}\right)} J_{i}\right\}^{2}\right]=\sum_{i=1}^{I}\left\{\frac{n_{i}^{4}}{\left(m_{i}\right)^{2}\left(n_{i}-m_{i}\right)^{2}}\right\}\left\{\frac{m_{i}\left(n_{i}-m_{i}\right)}{n_{i}} \sigma_{i, R}^{2}\right\}=s_{I}^{2}$

imsart-aoas ver. 2014/10/16 file: suppText-Revision3.tex date: November 10, 2015 
Then, by Theorem 9.2 in Chapter 9, Section 3 of Breiman (1992) (pg 187), the sum of $J_{i}$ weighted by $n_{i}^{2} / m_{i}\left(n_{i}-m_{i}\right)$ is a standard Normal distribution

$$
\frac{\sum_{i=1}^{I} \frac{n_{i}^{2}}{m_{i}\left(n_{i}-m_{i}\right)} J_{i}}{s_{I}} \rightarrow N(0,1)
$$

Fourth, for $H_{i}$, we have the following moments

$$
\begin{aligned}
E\left(H_{i}\right) & =\gamma m_{i}\left(1-\frac{m_{i}}{n_{i}}\right) \\
\operatorname{Var}\left(H_{i}\right) & =\operatorname{Var}\left(\sum_{j=1}^{n_{i}}\left(Z_{i j}-\bar{Z}_{i .}\right)\left(D_{i j}-\bar{D}_{i .}\right)\right) \\
& =\left(1-\frac{1}{n_{i}}\right) \sigma_{i, D}^{2} \sum_{j=1}^{n_{i}}\left(Z_{i j}-\bar{Z}_{i .}\right)^{2}-\frac{1}{n_{i}} \sigma_{i, D}^{2} \sum_{j, k}\left(Z_{i j}-\bar{Z}_{i .}\right)\left(Z_{i k}-\bar{Z}_{k .}\right) \\
& =\sigma_{i, D}^{2} \sum_{j=1}^{n_{i}}\left(Z_{i j}-\bar{Z}_{i .}\right)^{2}-\frac{1}{n_{i}} \sigma_{i, D}^{2}\left(\sum_{j=1}^{n_{i}}\left(Z_{i j}-\bar{Z}_{i .}\right)\right)^{2} \\
& =\sigma_{i, D}^{2} \frac{m_{i}\left(n_{i}-m_{i}\right)}{n_{i}}
\end{aligned}
$$

Fifth, by Theorem C in page 27 of Serfling (1980),

$$
\begin{aligned}
& \frac{1}{I} \sum_{i=1}^{I} \frac{n_{i}^{2}}{m_{i}\left(n_{i}-m_{i}\right)} H_{i}-\gamma \frac{1}{I} \sum_{i=1}^{I} E\left\{\frac{n_{i}^{2}}{m_{i}\left(n_{i}-m_{i}\right)} H_{i}\right\} \\
= & \frac{1}{I} \sum_{i=1}^{I} \frac{n_{i}^{2}}{m_{i}\left(n_{i}-m_{i}\right)} H_{i}-\gamma \frac{1}{I} \sum_{i=1}^{I} n_{i} \rightarrow 0
\end{aligned}
$$

Finally, combining all these facts together, we can rewrite the effect ratio estimator as follows.

$$
\begin{aligned}
\hat{\beta} & =\frac{\sum_{i=1}^{I} \frac{n_{i}^{2}}{m_{i}\left(n_{i}-m_{i}\right)} \sum_{j=1}^{n_{i}}\left(Z_{i j}-\bar{Z}_{i .}\right)\left(R_{i j}-\bar{R}_{i .}\right)}{\sum_{i=1}^{I} \frac{n_{i}^{2}}{m_{i}\left(n_{i}-m_{i}\right)} \sum_{j=1}^{n_{i}}\left(Z_{i j}-\bar{Z}_{i .}\right)\left(D_{i j}-\bar{D}_{i .}\right)} \\
& =\beta+\frac{\sum_{i=1}^{I} \frac{n_{i}^{2}}{m_{i}\left(n_{i}-m_{i}\right)} \sum_{j=1}^{n_{i}}\left(Z_{i j}-\bar{Z}_{i .}\right)\left(\epsilon_{i j}-\bar{\epsilon}_{i .}\right)}{\sum_{i=1}^{I} \frac{n_{i}^{2}}{m_{i}\left(n_{i}-m_{i}\right)} H_{i}} \\
& =\beta+\frac{\sum_{i=1}^{I} \frac{n_{i}^{2}}{m_{i}\left(n_{i}-m_{i}\right)} J_{i}}{\sum_{i=1}^{I} \frac{n_{i}^{2}}{m_{i}\left(n_{i}-m_{i}\right)} H_{i}}
\end{aligned}
$$

imsart-aoas ver. 2014/10/16 file: suppText-Revision3.tex date: November 10, 2015 
which leads to

$$
\sqrt{I}(\hat{\beta}-\beta)=\left\{\frac{\sum_{i=1}^{I} \frac{n_{i}^{2}}{m_{i}\left(n_{i}-m_{i}\right)} J_{i}}{s_{I}}\right\}\left\{\frac{\frac{1}{\sqrt{I}} s_{I}}{\frac{n_{i}^{2}}{I} \sum_{i=1}^{I} \frac{n_{i}}{m_{i}\left(n_{i}-m_{i}\right)} H_{i}}\right\}
$$

Finally, using Slutsky's Theorem, $\sqrt{I}(\hat{\beta}-\beta)$ converges to a Normal distribution with mean 0 and stated asymptotic variance.

Proposition 3 provides an easy way to compare between different types of full matching methods and their effect on the estimation of the effect ratio. For example, in the simple case of homoscedastic variance, the approximate variance of $\hat{\lambda}$ is

$$
\operatorname{Var}(\hat{\lambda}) \approx K \frac{\sum_{i=1}^{I} \frac{n_{i}^{3}}{n_{i}-1}}{\left(\sum_{i=1}^{I} n_{i}\right)^{2}}
$$

where $K$ is some constant that depends on the variance of $R_{i j}$ and the strength of the instrument. Since $K$ will be identical for all full matched designs, we can simply look at the quantities to the right of $K$ to tweak our full matching algorithm to produce the most efficient estimator.

With regards to the quality of the approximation, the asymptotic variance is a decent approximation to the estimator's variance if the number of matched sets, $I$, are large or if the instruments are strong. This is demonstrated in Table 1 which is a result of the following simulation study. The variables $R_{i j}, D_{i j}$ and $Z_{i j}$ are generated via the model in (12) and (13) with $Z_{i j}$ assumed to be fixed. We randomly pick $\alpha_{i}, \tau_{i}$, and $\beta$. We pick $\gamma$ to be 1 for the strong instrument case and -0.2 for the weak instrument case. We assume a homoscedastic variance for the error terms where all the $\sigma_{i, R}^{2}, \sigma_{i, D}^{2}$, and $\sigma_{i, R D}$ are the same for every $i$. We compute the effect ratio estimator, repeat this process 1000 times, and compute the simulated variance. The theoretical variance is calculated based on the formula provided in Proposition 3 .

Table 1 shows us that for strong instruments, the agreement between theoretical formula in Proposition 3 and simulation is quite good for all values of $I$. On the other hand, for weak instruments, there is substantial deviation between the theoretical variance and the simulated variance until $I$ is above 5000 .

6.2. Simulation to approximate efficiency. The prior section offers a formula to compute efficiency of various full matching schemes. However, for the formula to be valid, it requires, among other things, a linear model

imsart-aoas ver. 2014/10/16 file: suppText-Revision3.tex date: November 10, 2015 
TABLE 1

Comparison of simulated variance and theoretical variance for different strength of instruments and matched set number I.

I Theoretical Variance Simulated Variance

\begin{tabular}{lllll} 
& Strong & Weak & Strong & Weak \\
\hline 50 & 0.024 & 0.59 & 0.028 & 3224.30 \\
100 & 0.012 & 0.30 & 0.012 & 181.06 \\
110 & 0.011 & 0.27 & 0.012 & 2506.92 \\
500 & 0.0024 & 0.060 & 0.0025 & 2.05 \\
1000 & 0.0012 & 0.030 & 0.0012 & 0.037 \\
5000 & 0.00024 & 0.0060 & 0.00024 & 0.0063 \\
10000 & 0.00012 & 0.0030 & 0.00012 & 0.0030
\end{tabular}

between the outcome, $R_{i j}$ and the exposure $D_{i j}$. In our study where stunting, the outcome, is a binary variable and malaria, the exposure, is a whole number, it is unreasonable to assume that $R_{i j}$ is a linear function of $D_{i j}$.

In such cases, we propose a simulation study to analyze efficiency for different full matching schemes. As an illustration, consider our study with the effect of malaria on stunting. For each matching scheme, we fix $Z_{i j}$ and $X_{i j}$, which, in turn, fixes the matched sets. For the other variables, $D_{i j}$ and $R_{i j}$, we assume a Poisson relationship between $D_{i j}$ and $Z_{i j}$ and a logistic relationship between $D_{i j}$ and $R_{i j}$. In particular, we use the following model

$$
P\left(R_{i j}=1\right)=\frac{1}{1+e^{-\left(\alpha_{i}+\beta D_{i j}+u_{i j}\right)}}, \quad E\left(D_{i j}\right)=e^{\tau_{i}+\gamma Z_{i j}}
$$

We fix $\beta$, the effect of malaria on stunting, to be 0.32 and $\gamma$, the strength of the instrument, to be -0.20 based on the estimates in Kang et al. (2013); the estimate of $\gamma$ was based on the risk ratio estimate. We also randomly choose $\alpha_{i}$ and $\tau_{i}$, the intercepts, from Normal distributions with means -1.67 and -0.19 , respectively, and variances 0.12 and 0.027 , respectively. The mean and the variance for $\alpha_{i}$ is from the intercept term and its corresponding standard error of the logistic regression between $R_{i j}$ and $D_{i j}$. Similarly, the mean and the variance for $\tau_{i}$ is from the intercept term and its corresponding standard error of the Poisson regression between $D_{i j}$ and $Z_{i j}$. Once all the parameters are set, we sample 884 observations of $\left(R_{i j}, D_{i j}\right)$ (i.e. the sample size of the malaria data set) and compute the effect ratio estimator based on the sample of 884 . Note that the effect ratio estimator should be able to estimate $\beta$ since it doesn't rely on the functional form between stunting (i.e. outcome) and malaria episodes (i.e. exposure). We repeat the simulation 5000 times and compute the median absolute deviation as a robust proxy for variance of the effect ratio estimator.

Table 2 shows the trade-off between efficiency and covariate balance for

imsart-aoas ver. 2014/10/16 file: suppText-Revision3.tex date: November 10, 2015 
TABLE 2

Trade-off between efficiency and balance for different full matching schemes that use all the data based on simulation based on median absolute deviation and standardized bias.

Matching

Full matching (max strata size is 9)

Full matching (max strata size is 10 )

Full matching (max strata size is 15)

Full matching (unrestricted) Median absolute deviation

0.90

0.96

0.97

0.98
Standardized bias

0.23

0.19

0.10

0.055

different full matching schemes that use all 884 samples of the malaria data. In particular, we restrict the matched set sizes to different values to see their impact on efficiency and standardized bias. The standardized bias is the instrumental propensity score (Cheng, 2011) and is calculated as the difference in propensity scores before and after matching normalized by the within group standard deviation before matching (the square root of the average of the variances within the group). We see that unrestricted full matching has the lowest bias among all other full matching schemes. However, full matching with restricted strata size of 9 has the lowest median absolute deviation, albeit by a little in comparison to other matching schemes. Given the large bias reduction by using unrestricted full matching with a small gain in median absolute deviation, we use unrestricted full matching in our main manuscript.

\section{Supplementary Materials: Extended Simulation.}

7.1. Strength of instruments. The simulation setup is identical to the one in Section 3 of the main manuscript. We present another aspect of our estimator's performance in relation to 2SLS, specifically the median absolute deviation (MAD). Figure 2 measures the MAD of 2SLS and our method. Our method tends to have a slightly higher MAD than 2SLS. This higher variability of our method is to be expected since our method uses a nonparametric approach whereas 2SLS is a parametric approach. However, as the instrument gets stronger (i.e. high concentration parameter), the gap between the two MADs shrinks quickly.

7.2. Sample size. The simulation result presented here has the identical setup as the one in Section 3 of the main manuscript. However, we fix the strength of the instrument to be very strong, but vary the sample size. We keep the ratio between $Z_{i j}=1$ to $Z_{i j}=0$ to be 1 to 7 , respectively. We compare the performance of 2SLS and our method with respect to bias, variance, and type I error rate as we vary $f(\cdot)$.

imsart-aoas ver. 2014/10/16 file: suppText-Revision3.tex date: November 10, 2015 

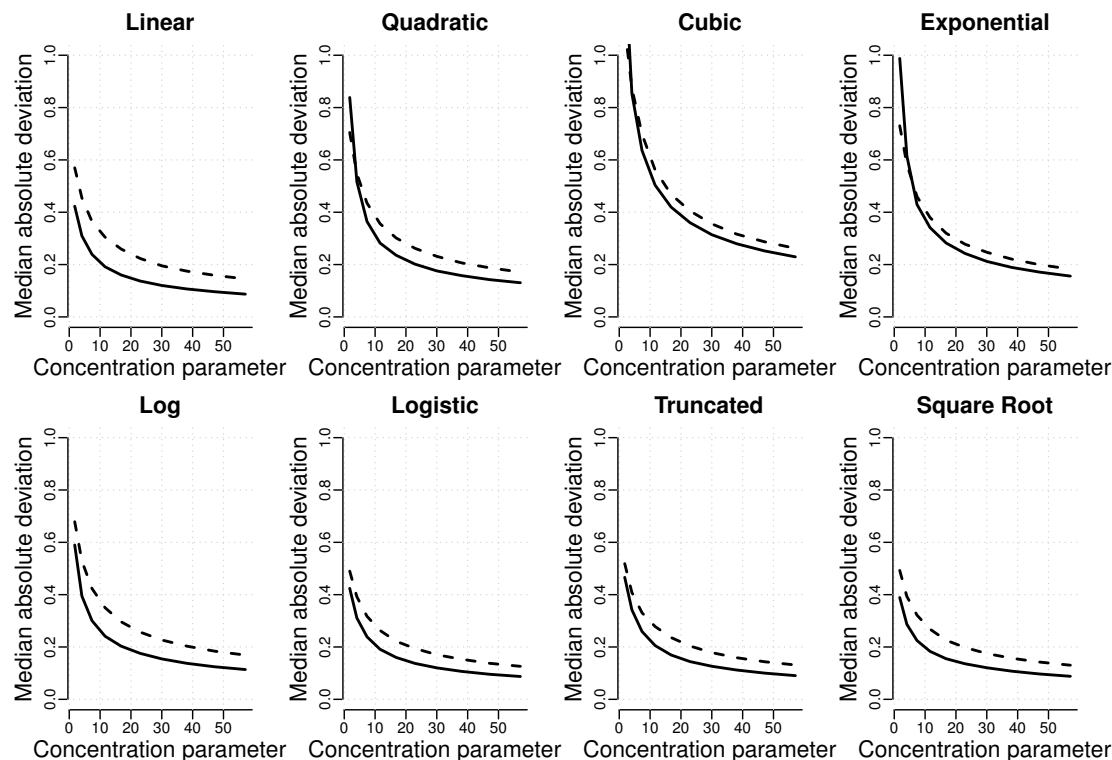

FIG 2. Median absolute deviation between our method and two stage least squares (2SLS) for different concentration parameters. The solid line indicates $2 S L S$ and the dotted line indicates our method. 

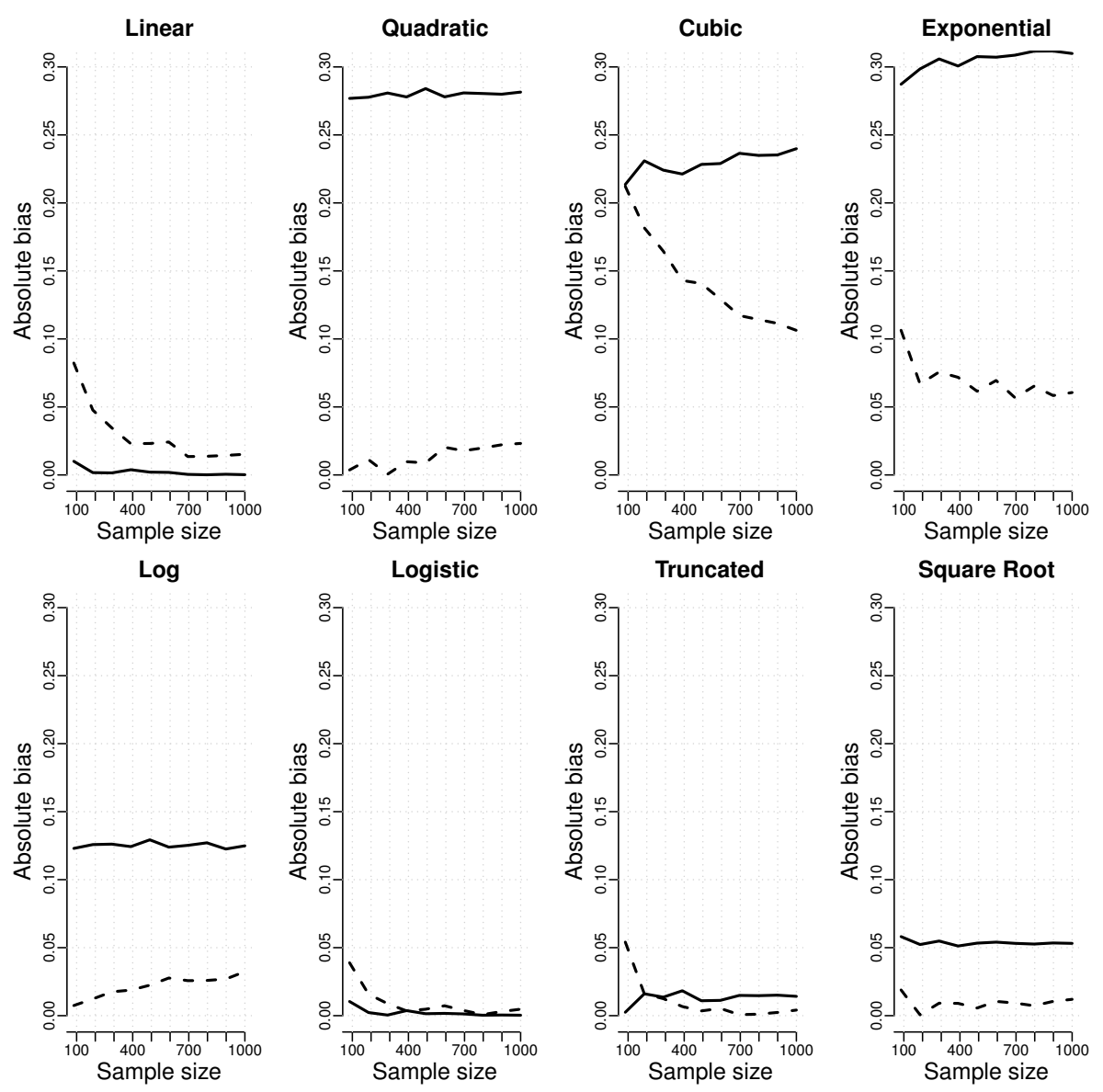

FIG 3. Absolute bias of the median between our method and two stage least squares (2SLS) for different sample sizes. The solid line indicates $2 S L S$ and the dotted line indicates our method.

imsart-aoas ver. 2014/10/16 file: suppText-Revision3.tex date: November 10, 2015 
Figure 3 measures the absolute bias of 2SLS and our method. When $f(\cdot)$ is a linear function of the observed covariates $\mathbf{x}_{i j}$, 2SLS does better than our method, which is to be expected since 2SLS works best when the model is linear. However, if $f(\cdot)$ is non-linear, our matching estimator does better than 2SLS and is never substantially worse. For example, for quadratic, cubic, exponential, log, and square root functions, our method has lower bias than 2SLS for all sample size. For logistic and truncated functions, our method is similar in performance to 2SLS.

Figure 4 measures the median absolute deviation (MAD) of 2SLS and our method. Our method tends to have a slightly higher MAD than 2SLS. This higher variability of our method is to be expected since our method uses a nonparametric approach whereas 2SLS is a parametric approach.

Finally, Figure 5 measures the Type I error rate of 2SLS and our method. Regardless of the function type and and sample size, our method retains the nominal 0.05 rate. In fact, even for the linear case where 2SLS is designed to excel, our estimator has the correct Type I error rate for all sample size while 2SLS has higher Type I error for small sample size. For all the nonlinear functions, the Type I error rate for 2SLS remains above the 0.05 line, with the notable exception of logistic and truncated functions whose 2SLS estimators has similar Type I error as our method. In contrast, our estimator maintains the nominal Type I error rate for all sample sizes. This provides evidence that our estimator will have the correct $95 \%$ coverage for confidence intervals regardless of the non-linearity or for different sample size.

7.3. Comparison to Frölich (2007). In this section, we provide a few additional details of our matching estimator to another non-parametric IV estimator with covariates explored by Frölich (2007) as presented in the main manuscript. The simulation setup is designed to mimic the data type in the malaria data where we have a binary $Z_{i j}$, discrete $D_{i j}$ taking on values 0,1 and 2 , and a continuous response $R_{i j}$ and is identical to the one presented in the main manuscript. Specifically, we have

$$
\begin{aligned}
& R_{i j}=\alpha+\beta D_{i j}+f\left(\mathbf{X}_{i j}\right)+U_{i j}+\epsilon_{i j} \\
& D_{i j}=\chi\left(D_{i j}^{*}<-1\right)+2 \chi\left(-1 \leq D_{i j}^{*}<1\right)+3 \chi\left(1 \leq D_{i j}^{*}\right) \\
& D_{i j}^{*}=\kappa+\pi Z_{i j}+\boldsymbol{\rho}^{T} \mathbf{X}_{i j}+U_{i j}+\xi_{i j}
\end{aligned}
$$

where $D_{i j}^{*}$ is a latent variable, $U_{i j}$ serve as the unmeasured confounder, and $\epsilon_{i j}, \xi_{i j}, U_{i j}$ are all i.i.d Normal. Covariates $X_{i j}$ are generated similar to the simulation study in the main manuscript. As before, we look at bias and variance across different strengths of instruments, different sample size, and different functions $f(\cdot)$. Similar to the main manuscript, we use the default

imsart-aoas ver. 2014/10/16 file: suppText-Revision3.tex date: November 10, 2015 

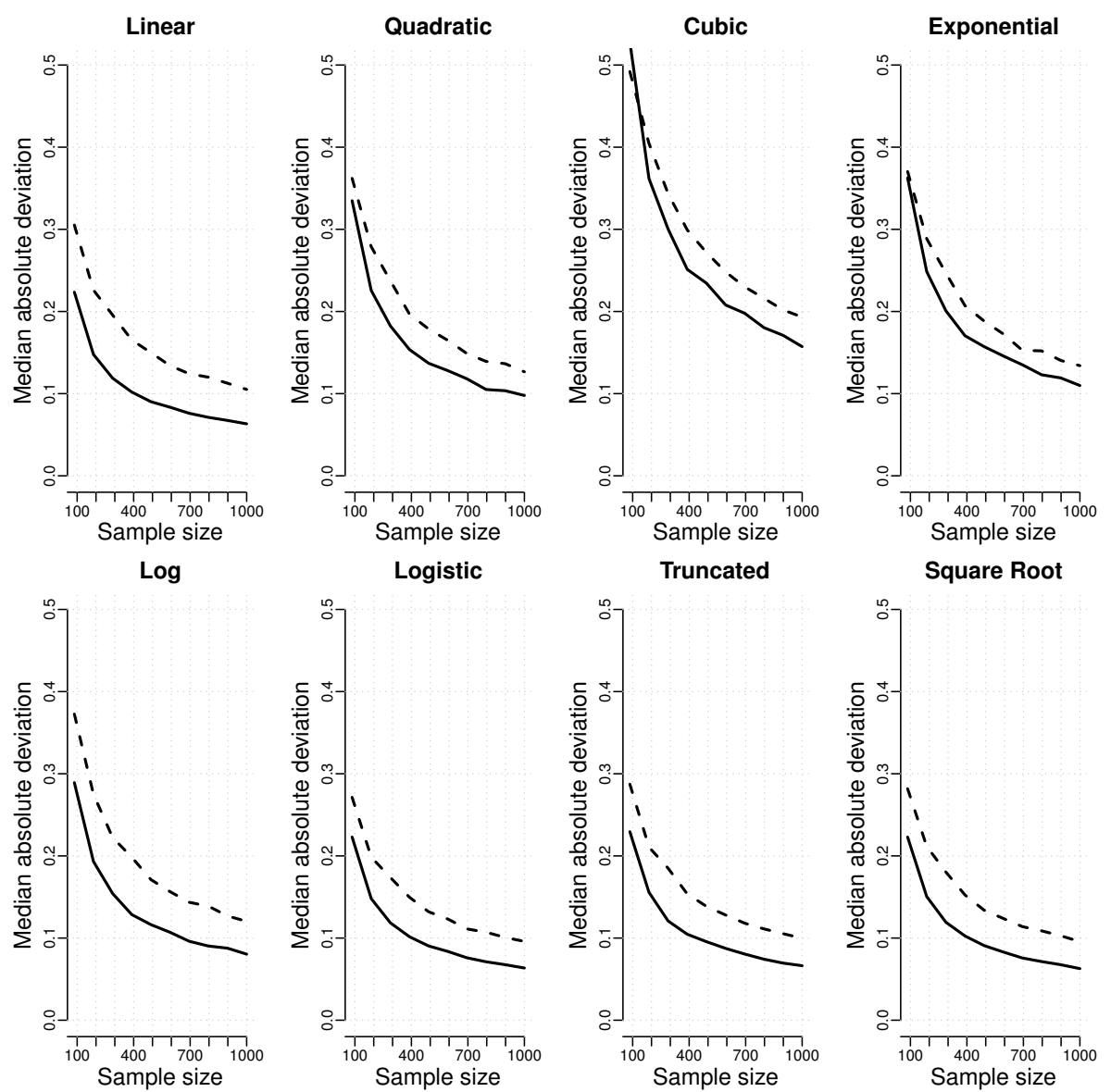

FIG 4. Median absolute deviation between our method and two stage least squares (2SLS) for different sample sizes. The solid line indicates 2SLS and the dotted line indicates our method. 

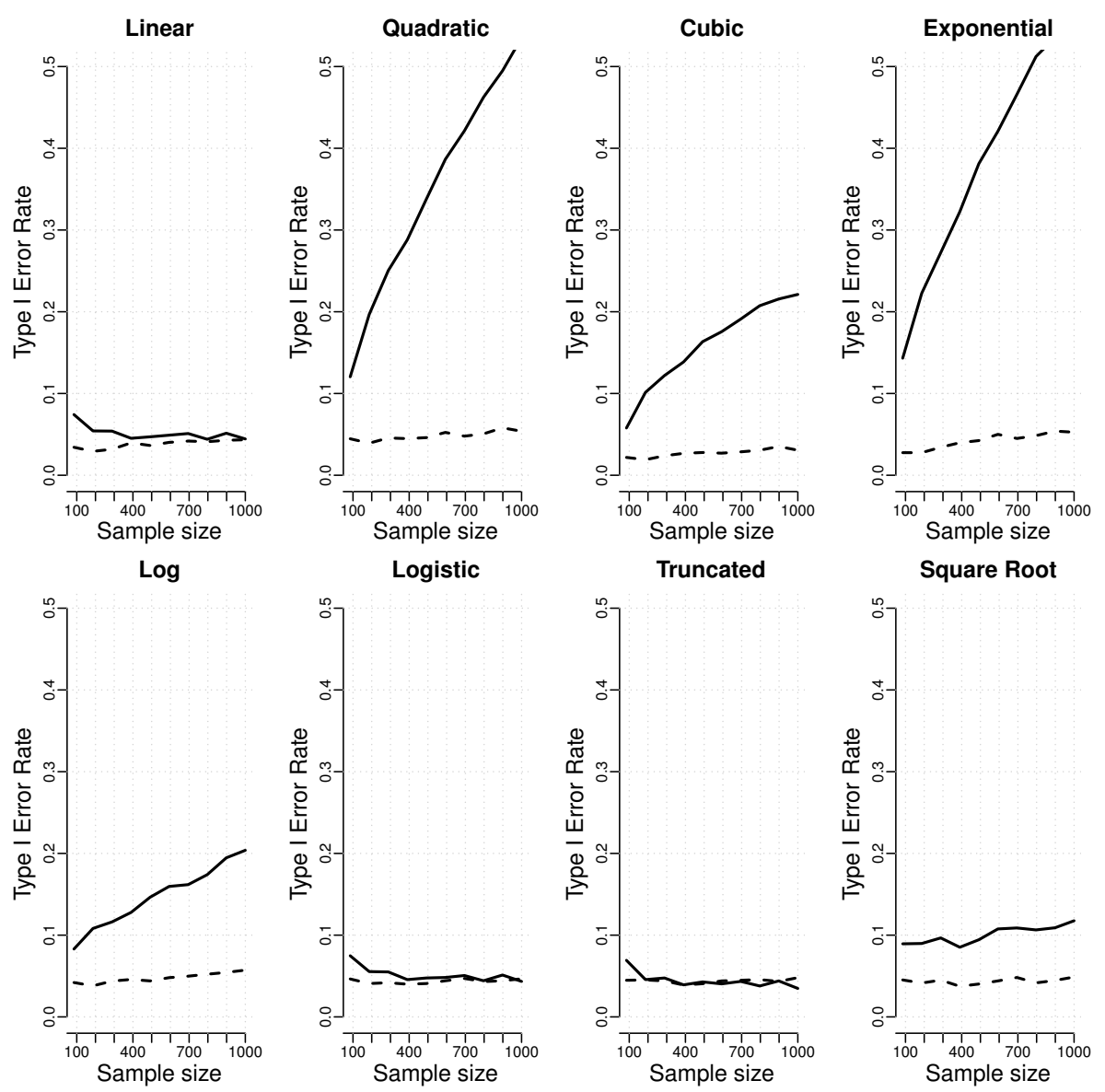

FIG 5. Type I error rate between our method and two stage least squares (2SLS) for different sample sizes. The solid line indicates $2 S L S$ and the dotted line indicates our method. 
settings provided in Frölich and Melly (2010), which implements the method by Frölich (2007).

We were not able to produce Type I error results for the method of Frölich (2007) because of a coding error in the code provided by Frölich and Melly (2010) which provided negative standard errors on the estimates produced by it. Frölich (personal communication) is aware of the issue and will be releasing a new version in the future.

\section{Supplementary Materials: Proof to Lemmas.}

Proof of LemMa 1. Let $y_{0 i j, \lambda_{0}}=r_{0 i j}^{\left(d_{0 i j}\right)}-\lambda_{0} d_{0 i j}$ and $y_{1 i j, \lambda_{0}}=r_{1 i j}^{\left(d_{1 i j}\right)}-$ $\lambda_{0} d_{1 i j}$. Then, $V_{i}\left(\lambda_{0}\right)$ becomes

$$
\begin{aligned}
V_{i}\left(\lambda_{0}\right) & =\frac{n_{i}}{m_{i}} \sum_{j=1}^{n_{i}} Z_{i j}\left(R_{i j}-\lambda_{0} D_{i j}\right)-\frac{n_{i}}{n_{i}-m_{i}} \sum_{j=1}^{n_{i}}\left(1-Z_{i j}\right)\left(R_{i j}-\lambda_{0} D_{i j}\right) \\
& =\frac{n_{i}}{m_{i}} \sum_{j=1}^{n_{i}} Z_{i j} y_{1 i j, \lambda_{0}}-\frac{n_{i}}{n_{i}-m_{i}} \sum_{j=1}^{n_{i}}\left(1-Z_{i j}\right) y_{0 i j, \lambda_{0}}
\end{aligned}
$$

By assumption (A3) of IV in the main manuscript, $Z_{i j}$ are independent within each strata. Then, for any $i=1, \ldots, I$ and for $j, k=1, \ldots, n_{i}$ where $j \neq k$

$$
E\left(Z_{i j} \mid \mathcal{F}, \mathcal{Z}\right)=\frac{m_{i}}{n_{i}}, \quad E\left(Z_{i j} Z_{i k} \mid \mathcal{F}, \mathcal{Z}\right)=\frac{m_{i}\left(m_{i}-1\right)}{n_{i}\left(n_{i}-1\right)}=\frac{m_{i}-1}{n_{i}}
$$

where the second equality is true because in full matching, $m_{i}=1$ and $n_{i}=m_{i}-1$ or $m_{i}=n_{i}-1$ and $n_{i}=1$. Then, the expectation of $V_{i}\left(\lambda_{0}\right)$ and the test statistic $T\left(\lambda_{0}\right)$ are

$$
\begin{aligned}
& E\left\{V_{i}\left(\lambda_{0}\right) \mid \mathcal{F}, \mathcal{Z}\right\}=\sum_{j=1}^{n_{i}}\left(r_{1 i j}^{\left(d_{1 i j}\right)}-r_{0 i j}^{\left(d_{0 i j}\right)}\right)-\lambda_{0}\left(d_{1 i j}-d_{0 i j}\right) \\
& E\left\{T\left(\lambda_{0}\right) \mid \mathcal{F}, \mathcal{Z}\right\}=\frac{1}{I} \sum_{i=1}^{I} E\left\{V_{i}\left(\lambda_{0}\right) \mid \mathcal{F}, \mathcal{Z}\right\}=\frac{1}{I}\left(\lambda-\lambda_{0}\right) \sum_{i=1}^{I} \sum_{j=1}^{n_{i}}\left(d_{1 i j}-d_{0 i j}\right)
\end{aligned}
$$

For variance of $V_{i}\left(\lambda_{0}\right)$, Proposition 2 in Rosenbaum (2002, Sec. 2.4.4) gives 
us

$$
\begin{aligned}
& \operatorname{Var}\left\{V_{i}\left(\lambda_{0}\right) \mid \mathcal{F}, \mathcal{Z}\right\} \\
= & \operatorname{Var}\left\{\sum_{j=1}^{n_{i}} Z_{i j}\left(\frac{n_{i}}{m_{i}} y_{1 i j, \lambda_{0}}+\frac{n_{i}}{n_{i}-m_{i}} y_{0 i j, \lambda_{0}}\right) \mid \mathcal{F}, \mathcal{Z}\right\} \\
= & \sum_{j=1}^{n_{i}}\left(\frac{m_{i}}{n_{i}}-\frac{m_{i}^{2}}{n_{i}^{2}}\right) a_{i j, \lambda_{0}}^{2}+\left(\frac{m_{i}-1}{n_{i}}-\frac{m_{i}^{2}}{n_{i}^{2}}\right) \sum_{j \neq k} a_{i j, \lambda_{0}} a_{i k, \lambda_{0}} \\
= & \left(\frac{m_{i}}{n_{i}}-\frac{m_{i}^{2}}{n_{i}^{2}}-\frac{m_{i}-1}{n_{i}}+\frac{m_{i}^{2}}{n_{i}^{2}}\right) \sum_{j=1}^{n_{i}} a_{i j, \lambda_{0}}^{2}+\left(\frac{m_{i}-1}{n_{i}}-\frac{m_{i}^{2}}{n_{i}^{2}}\right) \sum_{j, k} a_{i j, \lambda_{0}} a_{i k, \lambda_{0}} \\
= & \frac{1}{n_{i}} \sum_{j=1}^{n_{i}} a_{i j, \lambda_{0}}^{2}+\frac{n_{i}\left(m_{i}-1\right)-m_{i}^{2}}{n_{i}^{2}} \sum_{j, k} a_{i j, \lambda_{0}} a_{i k, \lambda_{0}} \\
= & \frac{1}{n_{i}} \sum_{j=1}^{n_{i}} a_{i j, \lambda_{0}}^{2}-\frac{1}{n_{i}^{2}} \sum_{j, k} a_{i j, \lambda_{0}} a_{i k, \lambda_{0}} \\
= & \frac{1}{n_{i}} \sum_{i=1}^{n_{i}}\left(a_{i j, \lambda_{0}}-\bar{a}_{i, \lambda}\right)^{2}
\end{aligned}
$$

Finally, the variance of $T\left(\lambda_{0}\right)$ is given by

$\operatorname{Var}\left\{T\left(\lambda_{0}\right) \mid \mathcal{F}, \mathcal{Z}\right\}=\frac{1}{D^{2}} \sum_{i=1}^{I} \operatorname{Var}\left\{V_{i}\left(\lambda_{0}\right) \mid \mathcal{F}, \mathcal{Z}\right\}=\frac{1}{I^{2}} \sum_{i=1}^{I} \frac{1}{n_{i}} \sum_{j=1}^{n_{i}}\left(a_{i j, \lambda_{0}}-\bar{a}_{i, \lambda}\right)^{2}$

Proof of Lemma 2. Let $v_{i, \lambda_{0}}^{2}=\operatorname{Var}\left\{V_{i}\left(\lambda_{0}\right) \mid \mathcal{F}, \mathcal{Z}\right\}$. Under the general- 
ized effect ratio, the bias of the estimator (3) is

$$
\begin{aligned}
& E\left\{S^{2}\left(\lambda_{0}\right) \mid \mathcal{F}, \mathcal{Z}\right\} \\
= & \frac{1}{I(I-1)} \sum_{i=1}^{I} E\left[\left\{V_{i}\left(\lambda_{0}\right)-T\left(\lambda_{0}\right)\right\}^{2} \mid \mathcal{F}, \mathcal{Z}\right] \\
= & \frac{1}{I(I-1)} \sum_{i=1}^{I} E\left\{V_{i}^{2}\left(\lambda_{0}\right) \mid \mathcal{F}, \mathcal{Z}\right\}+E\left\{T^{2}\left(\lambda_{0}\right) \mid \mathcal{F}, \mathcal{Z}\right\}-2 E\left\{V_{i}\left(\lambda_{0}\right) T\left(\lambda_{0}\right) \mid \mathcal{F}, \mathcal{Z}\right\} \\
= & \frac{1}{I(I-1)} \sum_{i=1}^{I}\left(\mu_{i, \lambda_{0}}^{2}+v_{i, \lambda_{0}}\right)+\left(\mu_{\lambda_{0}}^{2}+\frac{1}{I^{2}} \sum_{j=1}^{I} v_{j, \lambda_{0}}\right) \\
& -\frac{2}{I}\left(\mu_{i, \lambda_{0}}^{2}+v_{i, \lambda_{0}}+\sum_{j \neq i} \mu_{i, \lambda_{0}} \mu_{j, \lambda_{0}}\right) \\
= & \frac{1}{I(I-1)} \sum_{i=1}^{I}\left(v_{i, \lambda_{0}}-\frac{2}{I} v_{i, \lambda_{0}}+\frac{1}{I^{2}} \sum_{j=1}^{I} v_{j, \lambda_{0}}\right) \\
& +\frac{1}{I(I-1)} \sum_{i=1}^{I}\left(\mu_{i, \lambda_{0}}^{2}+\mu_{\lambda_{0}}^{2}-\frac{2}{I} \sum_{j=1}^{I} \mu_{i, \lambda_{0}} \mu_{j, \lambda_{0}}\right) \\
= & \left(\frac{I^{2}-2 I+I}{I(I-1)}\right) \frac{1}{I^{2}} \sum_{i=1}^{n} v_{i, \lambda_{0}}+\frac{1}{I(I-1)} \sum_{i=1}^{I}\left(\mu_{i, \lambda_{0}}-\mu_{\lambda_{0}}\right)^{2} \\
= & \frac{1}{I^{2}} \sum_{i=1}^{I} v_{i, \lambda_{0}}+\frac{1}{I(I-1)} \sum_{i=1}^{I}\left(\mu_{i, \lambda_{0}}-\mu_{\lambda_{0}}\right)^{2} \\
& \\
& \\
&
\end{aligned}
$$

\section{References.}

Serfling, R. (1980). Approximation Theorems of Mathematical Statistics New York: Wiley. Breiman, L. (1992). Probability: Classics in Applied Mathematics, vol. 7, pp. 186, Philadelphia: Society for Industrial and Applied Mathematics.

Cheng, J. (2011). Using the instrumental propensity score in observational studies for causal effects. Joint Statistical Meeting Presentation.

Frölich, M. (2007). Nonparametric IV estimation of local average treatment effects with covariates. Journal of Econometrics, 139, 35-75.

Frölich, M. and Melly, B. (2010). Estimation of quantile treatment effects with Stata. Stata Journal, 10, 423-457.

Gastwirth, J. L., Krieger, A. M. and Rosenbaum, P. R. (2000). Asymptotic separability in sensitivity analysis. Journal of the Royal Statistical Society: Series B (Statistical Methodology), 62, 545-555.

imsart-aoas ver. 2014/10/16 file: suppText-Revision3.tex date: November 10, 2015 
Hansen, B. B. (2004). Full matching in an observational study of coaching for the sat. Journal of the American Statistical Association, 99, 609-618.

Hayfield, T. and Racine, J. (2008). Nonparametric econometrics: the np package Journal of Statistical Software, 27, 1-32.

Hodges, J. L. and Lehmann, E. L. (1963). Estimation of location based on ranks Annals of Mathematical Statistics, 34, 598-611.

Hogg, R. V., McKean, J. W., and Craig, A. T. (2005). Introduction to Mathematical Statistics, 6th edn. New Jersey: Prentice Hall.

Kang, H., Kreuels, B., Adjei, O., Krumkamp, R., May, J. and Small, D. S. (2013). The causal effect of malaria on stunting: a mendelian randomization and matching approach. International Journal of Epidemiology, 42, 1390-1398.

Rosenbaum, P. R. (2002). Observational Studies, 2nd edn. New York: Springer-Verlag. Amplification of sensitivity analysis in matched observational studies. Journal of the American Statistical Association, 104, 1398-1405.

Small, D. S., Gastwirth, J. L., Krieger, A. M., Rosenbaum, P. R. (2009). Simultaneous sensitivity analysis for observational studies using full matching or matching with multiple controls Statistics and Its Interface, 2, 203-211.

Wooldridge, J. M. (2010). Econometric Analysis of Cross Section and Panel Data, 2nd edn. Cambridge: MIT Press.

Department of Statistics

The Wharton School

University of Pennsylvania

Philadelphia, PA, USA19104

E-MAIL: khyuns@wharton.upenn.edu dsmall@wharton.upenn.edu
Division of Tropical Medicine

I. Department of Internal Medicine

University Medical Centre

Hamburg, Eppendorf, Germany

E-MAIL: kreuels@bni-hamburg.de

Research Group Infectious Disease Epidemiology

Bernhard Nocht Institute for Tropical Medicine

Hamburg, Germany

E-MAIL: kreuels@bni-hamburg.de may@bni.uni-hamburg.de 